\title{
RADIUS PROBLEMS FOR FUNCTIONS ASSOCIATED WITH A NEPHROID DOMAIN
}

\author{
LATEEF AHMAD WANI ${ }^{\dagger}$ AND A. SWAMINATHAN ${ }^{\ddagger}$
}

\begin{abstract}
Let $\mathcal{S}_{N e}^{*}$ be the collection of all analytic functions $f(z)$ defined on the open unit disk $\mathbb{D}$ and satisfying the normalizations $f(0)=f^{\prime}(0)-1=0$ such that the quantity $z f^{\prime}(z) / f(z)$ assumes values from the range of the function $\varphi_{N e}(z):=1+z-z^{3} / 3, z \in \mathbb{D}$, which is the interior of the nephroid given by

$$
\left((u-1)^{2}+v^{2}-\frac{4}{9}\right)^{3}-\frac{4 v^{2}}{3}=0 .
$$

In this work, we find sharp $\mathcal{S}_{N e^{*}}^{*}$-radii for several geometrically defined function classes introduced in the recent past. In particular, $\mathcal{S}_{N e^{*}}^{*}$ radius for the starlike class $\mathcal{S}^{*}$ is found to be $1 / 4$. Moreover, radii problems related to the families defined in terms of ratio of functions are also discussed. Sharpness of certain radii estimates are illustrated graphically.
\end{abstract}

\section{Introduction}

Let $\mathcal{A}$ be the class of all analytic functions satisfying the conditions $f(0)=0$ and $f^{\prime}(0)=1$ in the open unit disc $\mathbb{D}:=\{z:|z|<1\}$. Clearly, for each $f \in \mathcal{A}$, the function $\mathcal{Q}_{f}(z): \mathbb{D} \rightarrow \mathbb{C}$ given by

$$
\mathcal{Q}_{f}(z):=\frac{z f^{\prime}(z)}{f(z)}
$$

is analytic and satisfies $\mathcal{Q}_{f}(0)=1$. Let $\mathcal{S} \subset \mathcal{A}$ be the family of univalent functions, and $\mathcal{S}^{*}(\alpha) \subset \mathcal{S}$ be the family of starlike functions of order $\alpha(0 \leq \alpha<1)$ given by the analytic characterization

$$
\mathcal{S}^{*}(\alpha):=\left\{f \in \mathcal{A}: \operatorname{Re}\left(\mathcal{Q}_{f}(z)\right)>\alpha\right\}
$$

Further, let us define the class $\mathcal{C}(\alpha)$ by the relation: $f \in \mathcal{C}(\alpha) \Longleftrightarrow z f^{\prime} \in \mathcal{S}^{*}(\alpha)$. The functions in $\mathcal{S}^{*}:=\mathcal{S}^{*}(0)$ and $\mathcal{C}:=\mathcal{C}(0)$ are, respectively, starlike and convex in $\mathbb{D}$. For functions $f$ and $g$ analytic on $\mathbb{D}$, we say that $f$ is subordinate to $g$, written $f \prec g$, if there exists an analytic function $w$ satisfying $w(0)=0$ and $|w(z)|<1$ such that $f(z)=g(w(z))$. Indeed, $f \prec g \Longrightarrow f(0)=g(0)$ and $f(\mathbb{D}) \subset g(\mathbb{D})$. Furthermore, if the function $g$ is univalent, then $f \prec g \Longleftrightarrow f(0)=g(0)$ and $f(\mathbb{D}) \subset g(\mathbb{D})$. By unifying several earlier results on subordination, Ma and Minda [12] introduced the function class $\mathcal{S}^{*}(\varphi)$ which, for brevity, we write as definition.

Definition 1.1. Let $\mathcal{S}^{*}(\varphi)$ denote the class of functions characterized as

$$
\mathcal{S}^{*}(\varphi):=\left\{f \in \mathcal{A}: \mathcal{Q}_{f}(z) \prec \varphi(z)\right\}
$$

where the analytic function $\varphi: \mathbb{D} \rightarrow \mathbb{C}$ is required to satisfy the following conditions:

(i) $\varphi(z)$ is univalent with $\operatorname{Re}(\varphi)>0$,

2010 Mathematics Subject Classification. 30C45, 30C80.

Key words and phrases. Starlike functions, Subordination, Radius problem, Bernoulli and Booth lemniscates, Cardioid, Nephroid. 
(ii) $\varphi(\mathbb{D})$ is starlike with respect to $\varphi(0)=1$,

(iii) $\varphi(\mathbb{D})$ is symmetric about the real line, and

(iv) $\varphi^{\prime}(0)>0$.

Evidently, for every such $\varphi, \mathcal{S}^{*}(\varphi)$ is always a subclass of the class of starlike functions $\mathcal{S}^{*}$, and $\mathcal{S}^{*}(\varphi)=\mathcal{S}^{*}$ for $\varphi(z)=(1+z) /(1-z)$. We call $\mathcal{S}^{*}(\varphi)$ as the Ma-Minda type starlike class associated with $\varphi$. Specializing the function $\varphi$ in (1.2) yields several important function classes. For instance, $\mathcal{S}^{*}(\alpha):=\mathcal{S}^{*}\left(\frac{1+(1-2 \alpha) z}{1-z}\right)$, the Janowski class $\mathcal{S}^{*}[A, B]:=$ $\mathcal{S}^{*}\left(\frac{1+A z}{1+B z}\right)$, where $-1 \leq B<A \leq 1$, and many more. This interesting approach of constructing starlike classes has attracted many researchers, as a result, several Ma-Minda type starlike classes have been introduced and studied recently by modifying the right side function $\varphi$ in (1.2) appropriately. Below we mention a few of the recently introduced MaMinda type classes which will be later used in our discussion.

(1) $\mathcal{S}_{L}^{*}:=\mathcal{S}^{*}(\sqrt{1+z})$. This class was first introduced by Sokół and Stankiewicz [23], and later discussed extensively by many authors, see [1, 3, 24, 25] and references therein. The function $\varphi_{L}(z):=\sqrt{1+z}$ maps $\mathbb{D}$ onto the region bounded by the right-loop of lemniscate of Bernoulli $\left|w^{2}-1\right|=1$.

(2) $\mathcal{S}_{R L}^{*}:=\mathcal{S}^{*}\left(\varphi_{R L}\right)$ with

$$
\varphi_{R L}(z):=\sqrt{2}-(\sqrt{2}-1) \sqrt{\frac{1-z}{1+2(\sqrt{2}-1) z}}, \quad z \in \mathbb{D},
$$

was introduced by Mendiratta et al. [14]. The function $\varphi_{R L}(z)$ maps $\mathbb{D}$ univalently onto the interior of left-loop of shifted lemniscate of Bernoulli $\left|(w-\sqrt{2})^{2}-1\right|=1$.

(3) $\mathcal{S}_{\mathbb{C}}^{*}:=\mathcal{S}^{*}\left(z+\sqrt{1+z^{2}}\right)$. Introduced by Raina and Sokół [17], the analytic function

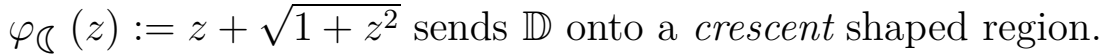

(4) $\mathcal{S}_{e}^{*}:=\mathcal{S}^{*}\left(e^{z}\right)$ was considered by Mendiratta et al. [15].

(5) $\mathcal{S}_{C}^{*}:=\mathcal{S}^{*}\left(1+4 z / 3+2 z^{2} / 3\right)$ was introduced by Sharma et al. [21]. The function $\varphi_{C}(z):=1+4 z / 3+2 z^{2} / 3$ sends $\mathbb{D}$ onto a region bounded by a cardioid.

(6) $\mathcal{S}_{R}^{*}:=\mathcal{S}^{*}\left(\varphi_{0}\right)$, where $\varphi_{0}(z)$ is the rational function

$$
\varphi_{0}(z):=1+\frac{z}{k}\left(\frac{k+z}{k-z}\right)=1+\frac{1}{k} z+\frac{2}{k^{2}} z^{2}+\cdots, \quad k=\sqrt{2}+1 .
$$

The class $\mathcal{S}_{R}^{*}$ was introduced by Kumar and Ravichandran [11].

(7) $\mathcal{B S}^{*}(\alpha):=\mathcal{S}^{*}\left(1+z /\left(1-\alpha z^{2}\right)\right), 0 \leq \alpha<1$, is the class associated with the Booth lemniscate which was introduced by Kargar et al. [9].

(8) $\mathcal{S}_{S}^{*}:=\mathcal{S}^{*}(1+\sin z)$ was introduced by Cho et al. [5]. The boundary of $\varphi_{S}(\mathbb{D})$, where $\varphi_{S}(z):=1+\sin z$, is an eight-shaped curve.

(9) For $0 \leq \alpha<1$, Khatter et al. [10] introduced

$$
\mathcal{S}_{\alpha, e}^{*}:=\mathcal{S}^{*}\left(\alpha+(1-\alpha) e^{z}\right) \text { and } \mathcal{S}_{L}^{*}(\alpha):=\mathcal{S}^{*}(\alpha+(1-\alpha) \sqrt{1+z}) .
$$

For $\alpha=0$, the above classes reduce to $\mathcal{S}_{e}^{*}$ and $\mathcal{S}_{L}^{*}$, respectively.

(10) Very recently, Wani and Swaminathan [27] introduced the function class

$$
\mathcal{S}_{N e}^{*}:=\mathcal{S}^{*}\left(1+z-z^{3} / 3\right) .
$$

The authors proved that the analytic function $\varphi_{N e}(z):=1+z-z^{3} / 3$ maps $\mathbb{D}$ univalently onto the interior of a 2-cusped kidney-shaped curve called nephroid given 
by

$$
\left((u-1)^{2}+v^{2}-\frac{4}{9}\right)^{3}-\frac{4 v^{2}}{3}=0 .
$$

Apart from studying several characteristic properties of the region bounded by the nephroid curve (1.4) (see Figure 1), the authors in [27] discussed certain inclusion relations, coefficient problems, and a few subordination results related to the function class $\mathcal{S}_{N e}^{*}$.

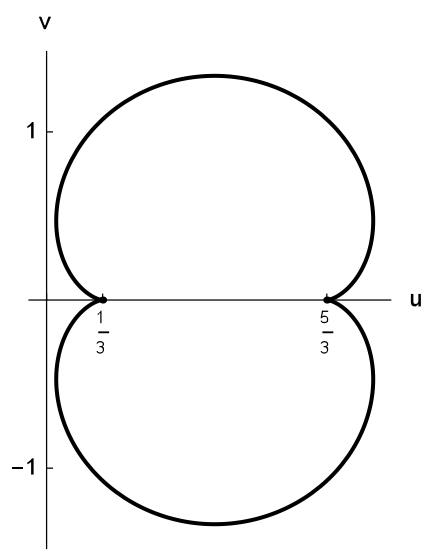

Figure 1. Nephroid: $\left((u-1)^{2}+v^{2}-4 / 9\right)^{3}-4 v^{2} / 3=0$.

\section{Radius Problems}

Let $\mathcal{F}$ and $\mathcal{G}$ be two subclasses of $\mathcal{A}$. Then the $\mathcal{F}$-radius for the class $\mathcal{G}$ is the largest number $\rho \in(0,1)$ such that $r^{-1} f(r z) \in \mathcal{F}$ for all $f \in \mathcal{G}$, where $0<r \leq \rho$. Moreover, the number $\rho$ is said to be sharp if there exists a function $f_{0} \in \mathcal{G}$ such that $r^{-1} f_{0}(r z) \notin \mathcal{F}$ whenever $r>\rho$ and such a function $f_{0}$ is called extremal function. The problem of finding the number " $\rho$ " is called a radius problem in geometric function theory (GFT). In the sequel, by

$$
R_{\mathcal{F}}(\mathcal{G})=\rho,
$$

we mean that $\rho$ is the $\mathcal{F}$-radius of $\mathcal{G}$. Below we present a table depicting several radii results related to some of the most important families in univalent function theory. For

\begin{tabular}{|c|c|c|c|}
\hline $\boldsymbol{R}_{\mathcal{F}}(\mathcal{G})$ & $\rho$ & $\begin{array}{l}\text { Approx. } \\
\text { value }\end{array}$ & Extremal function \\
\hline$R_{\mathcal{C}}(\mathcal{S})=R_{\mathcal{C}}\left(\mathcal{S}^{*}\right)$ & $2-\sqrt{3}$ & 0.267 & $\frac{z}{(1-z)^{2}}$ \\
\hline$R_{\mathcal{C}}\left(\mathcal{S}^{*}(1 / 2)\right)$ & $(2 \sqrt{3}-3)^{1 / 2}$ & 0.68125 & $\frac{z}{1-z}$ \\
\hline$R_{\mathcal{C}(\alpha)}\left(\mathcal{S}^{*}\right)$ & $\frac{2-\sqrt{3+\alpha^{2}}}{1+\alpha}$ & & $\frac{z}{(1-z)^{2}}$ \\
\hline$R_{\mathcal{C}(\alpha)}(\mathcal{C})$ & $\frac{1-\alpha}{1+\alpha}$ & & $\begin{array}{l}\frac{1-(1-z)^{2 \alpha-1}}{2 \alpha-1}, \text { if } \alpha \neq 1 / 2 \\
-\ln (1-z), \text { if } \alpha=1 / 2\end{array}$ \\
\hline
\end{tabular}
further details, we refer to [7, Chapter 13].

Table 1. Radii results of well-known classes 
Solving the radius problems for classes of functions with nice geometries is continuing to be an active area of research in GFT. Investigating the radii problems became a bit easier after the dawn of subordination theory, particularly in the cases where the class $\mathcal{F}$ has a Ma-Minda type representation (1.2). For such classes, we redefine the radius problem in geometrical terms as:

Definition 2.1. Let $\mathbb{D}_{r}:=\{z:|z|<r\}$ and $\Omega_{\varphi}:=\varphi(\mathbb{D})$. By $\mathcal{S}^{*}(\varphi)$-radius for the class $\mathcal{G} \subset \mathcal{A}$, denoted by $R_{\mathcal{S}_{\varphi}^{*}}(\mathcal{G})$, we mean the largest number $\rho \in(0,1)$ such that each $f \in \mathcal{G}$ satisfies

$$
\mathcal{Q}_{f}\left(\mathbb{D}_{r}\right) \subset \Omega_{\varphi} \text { for every } r \leq \rho,
$$

where $\mathcal{Q}_{f}(z)$ is defined in (1.1). Moreover, the number $\rho$ is said to be sharp if we can find a function $f_{0} \in \mathcal{G}$ such that

$$
\mathcal{Q}_{f_{0}}\left(\partial \mathbb{D}_{\rho}\right) \cap \partial \Omega_{\varphi} \neq \emptyset .
$$

For certain recent results in this direction, we provide the following table along with

\begin{tabular}{|c|c|c|c|c|}
\hline $\boldsymbol{R}_{\mathcal{S}_{\varphi}^{*}}(\mathcal{G})$ & $\rho$ & $\begin{array}{l}\text { Approx. } \\
\text { value }\end{array}$ & $\begin{array}{l}\text { Extremal } \\
\text { function }\end{array}$ & Refer \\
\hline$R_{\mathcal{S}_{(}^{*}}\left(\mathcal{S}^{*}\right)$ & $\sqrt{2}-1$ & 0.41421 & $\frac{z}{(1-z)^{2}}$ & 6 \\
\hline$R_{\mathcal{S}_{R L}^{*}}\left(\mathcal{S}^{*}\right)$ & $\sqrt{(\sqrt{5}-2)(5 \sqrt{2}-7)}$ & 0.129525 & $"$ & 14 \\
\hline$R_{\mathcal{S}_{e}^{*}}\left(\mathcal{S}^{*}\right)$ & $\frac{e-1}{e+1}$ & 0.462117 & $"$ & 15 \\
\hline$R_{\mathcal{S}_{(\mathcal{C}}^{*}}(\mathcal{C})$ & $2-\sqrt{2}$ & 0.58578 & $\frac{z}{1-z}$ & 6 \\
\hline$R_{\mathcal{S}_{e}^{*}}(\mathcal{C})$ & $\frac{e-1}{e}$ & 0.632121 & $"$ & [15] \\
\hline$R_{\mathcal{S}_{S}^{*}}\left(\mathcal{S}_{L}^{*}\right)$ & $(2-\sin 1) \sin 1$ & 0.975 & $\frac{4 z \exp (2 \sqrt{1+z}-2)}{1+\sqrt{1+z}}$ & [5] \\
\hline$R_{\mathcal{S}_{R}^{*}}\left(\mathcal{S}_{L}^{*}\right)$ & $8 \sqrt{2}-11$ & 0.313708 & $"$ & [1] \\
\hline$R_{\mathcal{S}^{*}(\alpha)}\left(\mathcal{S}_{L}^{*}\right)$ & $1-\alpha^{2}$ & & $"$ & [24] \\
\hline$R_{\mathcal{S}_{(}^{*}}\left(\mathcal{S}_{e}^{*}\right)$ & $\log \left(\frac{\sqrt{2}+1}{2}\right)$ & 0.188226 & $z \exp \left(\int_{0}^{z} \frac{e^{t}-1}{t} d t\right)$ & 6 \\
\hline$R_{\mathcal{S}_{S}^{*}}\left(\mathcal{S}_{C}^{*}\right)$ & $\frac{1}{2}(\sqrt{2(2+3 \sin 1)}-2)$ & 0.504 & $z \exp \left(\frac{4 z}{3}+\frac{z^{2}}{3}\right)$ & 5 \\
\hline$R_{\mathcal{S}_{L}^{*}}\left(\mathcal{S}_{C}^{*}\right)$ & $-1+\sqrt{-\frac{1}{2}+\frac{3}{\sqrt{2}}}$ & 0.273311 & $"$ & 21 \\
\hline$R_{\mathcal{B S}^{*}(\alpha)}\left(\mathcal{S}_{S}^{*}\right)$ & $\sinh ^{-1}\left(\frac{1}{1+\alpha}\right)$ & & $z \exp \left(\int_{0}^{z} \frac{\sin t}{t} d t\right)$ & 4 \\
\hline$R_{\mathcal{S}_{R}^{*}}\left(\mathcal{S}_{S}^{*}\right)$ & $\log (3-2 \sqrt{2})+\sqrt{6}(\sqrt{2}-1)$ & 0.170742 & $"$ & 11 \\
\hline
\end{tabular}
the respective references. Also, see [1, 2, 10, 18] and the references therein.

Table 2. Some recent radii results 
Motivated by the aforementioned works, in this work, we consider the function class $\mathcal{S}_{N e}^{*}$ introduced by the authors in [27] and find sharp $\mathcal{S}_{N e^{*}}^{*}$-radii estimates for certain geometrically defined function classes available in the literature. More explicitly, in Section 3 , the $\mathcal{S}_{N e}^{*}$-radius problem is discussed for some of the recently introduced Ma-Minda type starlike classes. In particular, the Janowski class $\mathcal{S}^{*}[A, B]$ is considered, and consequently, the best possible $\mathcal{S}_{N e^{*}}^{*}$ radii for $\mathcal{S}^{*}$ and $\mathcal{C}$ are obtained as special cases. Finally, in Section 4, we consider several function families defined by the ratio of analytic functions and calculate $\mathcal{S}_{N e, n}^{*}$-radius for them, where

$$
\mathcal{S}_{N e, n}^{*}:=\mathcal{S}_{N e}^{*} \cap \mathcal{A}_{n},
$$

and $\mathcal{A}_{n}$ consists of analytic functions of the form $f(z)=z+\sum_{k=n+1}^{\infty} a_{k} z^{k}$. Apart from proving the sharpness of radii estimates analytically, certain graphical illustrations are also provided.

The following lemma related to the largest disks contained in the region bounded by the nephroid curve (1.4) is instrumental in proving our results.

Lemma 2.1. Let $1 / 3<a<5 / 3$, and let $r_{a}$ be given by

$$
r_{a}= \begin{cases}a-1 / 3 & \text { if } 1 / 3<a \leq 1 \\ 5 / 3-a & \text { if } 1 \leq a<5 / 3\end{cases}
$$

Then

$$
\left\{w \in \mathbb{C}:|w-a|<r_{a}\right\} \subseteq \Omega_{N e},
$$

where $\Omega_{N e}:=\varphi_{N e}(\mathbb{D})$, the region bounded by the nephroid (1.4).

Proof. For $z=e^{i t}$, the parametric equations of the nephroid $\varphi_{N e}(z)=1+z-z^{3} / 3$ are

$$
u(t)=1+\cos t-(\cos 3 t) / 3, \quad v(t)=\sin t-(\sin 3 t) / 3, \quad t \in(-\pi, \pi] .
$$

Let $a \in(1 / 3,5 / 3)$. Then the squared distance from the point $(a, 0)$ on the real line to the points on the nephroid curve (1.4) is given by

$$
\begin{aligned}
\xi(t) & =(a-u(t))^{2}+(v(t))^{2} \\
& =\frac{16}{9}+(a-1)^{2}-4(a-1) \cos t-\frac{4}{3} \cos ^{2} t+\frac{8}{3}(a-1) \cos ^{3} t \\
& =\frac{16}{9}+(a-1)^{2}-4(a-1) x-\frac{4}{3} x^{2}+\frac{8}{3}(a-1) x^{3}=: H(x),
\end{aligned}
$$

where $x=\cos t \in[-1,1],-\pi<t \leq \pi$. Since the nephroid curve (1.4) is symmetric about the real line, it is enough to consider $t \in[0, \pi]$. A simple computation shows that $H^{\prime}(x)=0$ if, and only if,

$$
x=x_{0}:=\frac{1-\sqrt{1+18(a-1)^{2}}}{6(a-1)} \text { and } x=x_{1}:=\frac{1+\sqrt{1+18(a-1)^{2}}}{6(a-1)} .
$$

For $a \in(1 / 3,5 / 3)$, further verification reveals (also see the plots in Figure 2) that only the number $x_{0}$ lies between -1 and 1 . Moreover,

$$
H^{\prime \prime}\left(x_{0}\right)=-\frac{8}{3} \sqrt{1+18(a-1)^{2}}<0 \text { for each } a .
$$

This shows that $x_{0}$ is the point of maxima for the function $H(x)$ and hence, $H(x)$ is increasing in the interval $\left[-1, x_{0}\right]$ and decreasing in $\left[x_{0}, 1\right]$. Thus, for $1 / 3<a<5 / 3$, we 
have

$$
\min _{0 \leq t \leq \pi} \xi(t)=\min \{H(-1), H(1)\}
$$
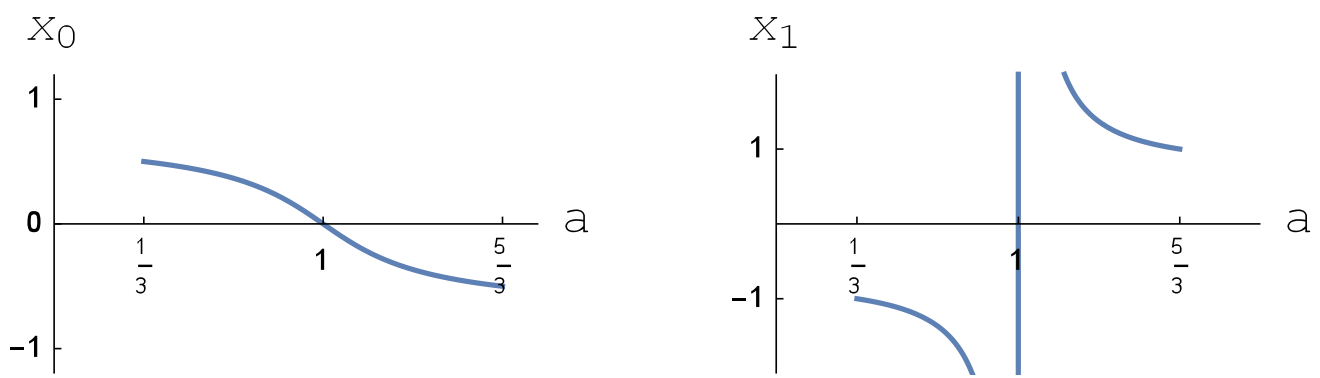

Figure 2. Plots of $x_{0}=x_{0}(a)$ and $x_{1}=x_{1}(a)$ for $a \in(1 / 3,5 / 3)$.

Also,

$$
H(1)-H(-1)=-8(a-1) / 3,
$$

so that $H(-1) \leq H(1)$ whenever $a \leq 1$, and $H(1) \leq H(-1)$ whenever $a \geq 1$. Therefore, we conclude that

$$
r_{a}=\min _{0 \leq t \leq \pi} \sqrt{\xi(t)}= \begin{cases}\sqrt{H(-1)}=a-1 / 3 & \text { whenever } 1 / 3<a \leq 1, \\ \sqrt{H(1)}=5 / 3-a & \text { whenever } 1 \leq a<5 / 3 .\end{cases}
$$

Thus, the disk $\left\{w \in \mathbb{C}:|w-a|<r_{a}\right\}$ completely lies inside the region $\Omega_{N e}$, and establishes the lemma.

\section{3. $\mathcal{S}_{N e}^{*}$-Radius For Ma-Minda Type Classes}

For $-1 \leq B<A \leq 1$, let $\mathcal{P}[A, B]$ be the collection of analytic functions $p: \mathbb{D} \rightarrow$ $\mathbb{C}$ that are of the form $p(z)=1+\sum_{n=1}^{\infty} p_{n} z^{n}$ and satisfy the subordination $p(z) \prec$ $(1+A z) /(1+B z)$. Also, recall [8] that the Janowski starlike class $\mathcal{S}^{*}[A, B]$ consists of functions $f \in \mathcal{A}$ satisfying $\mathcal{Q}_{f}(z) \in \mathcal{P}[A, B]$. To prove the results we use the following lemma.

Lemma 3.1 ([18, Lemma 2.1, p. 267]). If $p \in \mathcal{P}[A, B]$, then for $|z|=r<1$,

$$
\left|p(z)-\frac{1-A B r^{2}}{1-B^{2} r^{2}}\right| \leq \frac{(A-B) r}{1-B^{2} r^{2}}
$$

Theorem 3.1. (i) Let $0 \leq B<A \leq 1$. Then the $\mathcal{S}_{N e}^{*}$-radius for $\mathcal{S}^{*}[A, B]$ is given by

$$
R_{\mathcal{S}_{N e}^{*}}\left(\mathcal{S}^{*}[A, B]\right)=\min \left\{1, \frac{2}{3 A-B}\right\} \text {. }
$$

In particular, if $1-B \leq 3(1-A)$, then $\mathcal{S}^{*}[A, B] \subset \mathcal{S}_{N e}^{*}$.

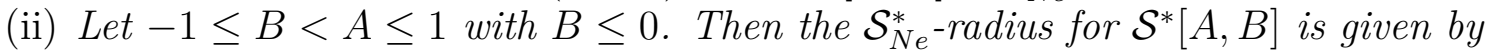

$$
R_{\mathcal{S}_{N e}^{*}}\left(\mathcal{S}^{*}[A, B]\right)=\min \left\{1, \frac{2}{3 A-5 B}\right\} .
$$

In particular, if $3(1+A) \leq 5(1+B)$, then $\mathcal{S}^{*}[A, B] \subset \mathcal{S}_{N e}^{*}$.

Proof. (i): Let $f \in \mathcal{S}^{*}[A, B]$. Then $\mathcal{Q}_{f} \in \mathcal{P}[A, B]$, and Lemma 3.1 yields

$$
\left|\mathcal{Q}_{f}(z)-\frac{1-A B r^{2}}{1-B^{2} r^{2}}\right| \leq \frac{(A-B) r}{1-B^{2} r^{2}} .
$$


The inequality (3.1) represents a disk with center at $\left(1-A B r^{2}\right) /\left(1-B^{2} r^{2}\right)$ and radius $(A-B) r /\left(1-B^{2} r^{2}\right)$. Since $B \geq 0$, we have $\left(1-A B r^{2}\right) /\left(1-B^{2} r^{2}\right) \leq 1$. Thus, by Lemma 2.1, the disk (3.1) lies completely inside the region $\Omega_{N e}$ bounded by the nephroid curve (1.4) if

$$
\frac{(A-B) r}{1-B^{2} r^{2}} \leq \frac{1-A B r^{2}}{1-B^{2} r^{2}}-\frac{1}{3}
$$

Simplifying the above inequality, we obtain $r \leq 2 /(3 A-B)=: \rho_{J}$. For sharpness of the estimate, consider the function

$$
f_{J}(z):= \begin{cases}z(1+B z)^{\frac{A-B}{B}}, & \text { if } B \neq 0 \\ z e^{A z}, & \text { if } B=0 .\end{cases}
$$

The function $f_{J}(z)$ satisfies $\mathcal{Q}_{f_{J}}(z)=(1+A z) /(1+B z)$, implying that $f_{J} \in \mathcal{S}^{*}[A, B]$. Also, for $z_{0}=-2 /(3 A-B) \in \partial \mathbb{D}_{\rho_{J}}$, we have

$$
\mathcal{Q}_{f_{J}}\left(z_{0}\right)=1 / 3 \in \partial \Omega_{N e}
$$

i.e.,

$$
\mathcal{Q}_{f_{J}}\left(\partial \mathbb{D}_{\rho_{J}}\right) \cap \partial \Omega_{N e}=\{1 / 3\} \neq \emptyset .
$$

This proves that the result is sharp and $f_{J} \in \mathcal{S}^{*}[A, B]$ is the extremal function. In particular, if $1-B \leq 3(1-A)$, then $2 /(3 A-B) \geq 1$ and hence, $R_{\mathcal{S}_{N e}^{*}}\left(\mathcal{S}^{*}[A, B]\right)=$ 1. That is, $\mathcal{S}^{*}[A, B] \subset \mathcal{S}_{N e}^{*}$.

(ii): As afore, $f \in \mathcal{S}^{*}[A, B]$ implies the inequality (3.1). Now, $B \leq 0$ implies that $\left(1-A B r^{2}\right) /\left(1-B^{2} r^{2}\right) \geq 1$. Therefore, in this case, the disk (3.1) lies in the interior of $\Omega_{N e}$ if

$$
\frac{(A-B) r}{1-B^{2} r^{2}} \leq \frac{5}{3}-\frac{1-A B r^{2}}{1-B^{2} r^{2}}
$$

Solving, we get $r \leq 2 /(3 A-5 B)$. Choosing $z_{0}=2 /(3 A-5 B)$ and $f_{J} \in \mathcal{S}^{*}[A, B]$ defined above, it can be easily verified that $\mathcal{Q}_{f_{J}}\left(z_{0}\right)=5 / 3 \in \partial \Omega_{N e}$. Hence, the result is sharp.

Let $0 \leq B<A \leq 1$. Using a result proved by Wani and Swaminathan [27], the following remark also shows that $1-B \leq 3(1-A)$ is indeed a sufficient condition for the inclusion $\mathcal{S}^{*}[A, B] \subset \mathcal{S}_{N e}^{*}$.

Remark 1. Since, $1-B \leq 3(1-A)$ implies $1-B^{2} \leq 3(1-A B+(B-A))<3(1-A B)$ and, $0 \leq B<A \leq 1$ gives $3(1-A B) \leq 3\left(1-B^{2}\right)$. Combining, we obtain $1-B^{2}<$ $3(1-A B) \leq 3\left(1-B^{2}\right)$. Now the inclusion relation $\mathcal{S}^{*}[A, B] \subset \mathcal{S}_{N e}^{*}$ follows from [27, Theorem 3.3].

Specializing the constants $A$ and $B$ in Theorem 3.1 , the following important sharp radii results are obtained.


The estimate is sharp for the function $k_{\alpha}(z)=z(1-z)^{2 \alpha-2}, 0 \leq \alpha<1$.

Corollary 3.2. The sharp $\mathcal{S}_{N e}^{*}$-radius for the class of starlike functions $\mathcal{S}^{*}$ is $1 / 4$, and the sharpness holds for the well-known Koebe function $k(z):=z /(1-z)^{2}$.

Corollary 3.3. The sharp $\mathcal{S}_{N e}^{*}$-radius for the convex class $\mathcal{C}$ is $2 / 5$. 
Proof. From Theorem [3.1, the $\mathcal{S}_{N e}^{*}$-radius for $\mathcal{S}^{*}(1 / 2)=\mathcal{S}^{*}[0,-1]$ is $2 / 5$. Using the fact that $\mathcal{C} \subset \mathcal{S}^{*}(1 / 2)$ (see [16, Theorem 2.6a, p. 57]), it follows that $R_{\mathcal{S}_{N e}^{*}}(\mathcal{C}) \geq 2 / 5$. Further, at the point $z_{0}=2 / 5 \in \partial \mathbb{D}_{2 / 5}$, the function $h(z):=z /(1-z) \in \mathcal{C}$ satisfies

$$
\mathcal{Q}_{h}\left(z_{0}\right)=1 /(1-2 / 5)=5 / 3 \in \partial \Omega_{N e},
$$

showing that $R_{\mathcal{S}_{N e}^{*}}(\mathcal{C}) \leq 2 / 5$. Hence, $R_{\mathcal{S}_{N e}^{*}}(\mathcal{C})=2 / 5$, and the estimate is sharp for the function $h(z)$.

Before proving the next result, we note that $\mathcal{S}_{L}^{*}(\alpha) \subset \mathcal{S}_{N e}^{*}$ for $\alpha \geq 1 / 3$, and $\mathcal{S}_{\alpha, e}^{*} \subset \mathcal{S}_{N e}^{*}$ for $\alpha \geq(3 e-5) /(3 e-3)$, (see Wani and Swaminathan [27, Theorem 3.1]). Therefore

$$
R_{\mathcal{S}_{N e}^{*}}\left(\mathcal{S}_{L}^{*}(\alpha)\right)=1, \text { whenever } \alpha \geq 1 / 3
$$

and

$$
R_{\mathcal{S}_{N e}^{*}}\left(\mathcal{S}_{\alpha, e}^{*}\right)=1 \text {, whenever } \alpha \geq(3 e-5) /(3 e-3) .
$$

Theorem 3.2. For the function classes $\mathcal{B S}^{*}(\alpha), \mathcal{S}_{L}^{*}(\alpha)$, and $\mathcal{S}_{\alpha, e}^{*}$, the following radius results hold:

(i) For $\alpha \in[0,1), R_{\mathcal{S}_{N e}^{*}}\left(\mathcal{B S}^{*}(\alpha)\right)=\rho_{\mathcal{B}}(\alpha):=4 /(3+\sqrt{9+16 \alpha})$.

(ii) For $\alpha \in[0,1 / 3], R_{\mathcal{S}_{N e}^{*}}\left(\mathcal{S}_{L}^{*}(\alpha)\right)=\rho_{L}(\alpha):=4(2-3 \alpha) / 9(1-\alpha)^{2}$. In particular, $R_{\mathcal{S}_{N e}^{*}}\left(\mathcal{S}_{L}^{*}\right)=\rho_{L}:=8 / 9$.

(iii) For $\alpha \in[0,(3 e-5) /(3 e-3)], R_{\mathcal{S}_{N e}^{*}}\left(\mathcal{S}_{\alpha, e}^{*}\right)=\rho_{e}(\alpha):=\log ((5-3 \alpha) /(3-3 \alpha))$. In particular, $R_{\mathcal{S}_{N e}^{*}}\left(\mathcal{S}_{e}^{*}\right)=\rho_{e}:=\log (5 / 3) \approx 0.510826$.

Each of the estimates is best possible.

Proof. (i): Let $f \in \mathcal{B S}^{*}(\alpha)$. Then $\mathcal{Q}_{f}(z) \prec G_{\alpha}(z):=1+z /\left(1-\alpha z^{2}\right)$ and hence, for $|z|=r$, we have

$$
\left|\mathcal{Q}_{f}(z)-1\right| \leq\left|z /\left(1-\alpha z^{2}\right)\right| \leq r /\left(1-\alpha r^{2}\right)
$$

In view of Lemma 2.1, the disk (3.2) lies completely inside the nephroid region $\Omega_{N e}$ if $r /\left(1-\alpha r^{2}\right) \leq 2 / 3$. This gives $r \leq \rho_{\mathcal{B}}(\alpha)$. For sharpness, consider the function

$$
f_{\mathcal{B}}(z)= \begin{cases}z\left(\frac{1+\sqrt{\alpha} z}{1-\sqrt{\alpha} z}\right)^{1 /(2 \sqrt{\alpha})}, & \alpha \in(0,1) \\ z e^{z}, & \alpha=0 .\end{cases}
$$

It is easy to verify that $\mathcal{Q}_{f_{\mathcal{B}}}(z)=G_{\alpha}(z)$, and hence $f_{\mathcal{B}} \in \mathcal{B S}^{*}(\alpha)$. Also, a straightforward calculation shows that

$$
\mathcal{Q}_{f_{\mathcal{B}}}\left(z_{0}\right)=1 / 3 \in \partial \Omega_{N e}, \text { for } z_{0}=-\rho_{\mathcal{B}}(\alpha),
$$

and

$$
\mathcal{Q}_{f_{\mathcal{B}}}\left(z_{1}\right)=5 / 3 \in \partial \Omega_{N e}, \text { for } z_{1}=\rho_{\mathcal{B}}(\alpha)
$$




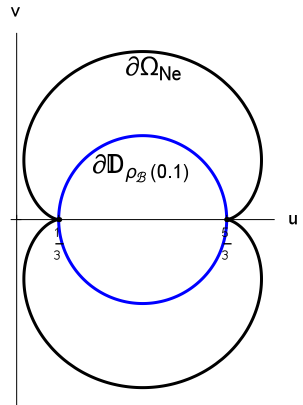

(a)

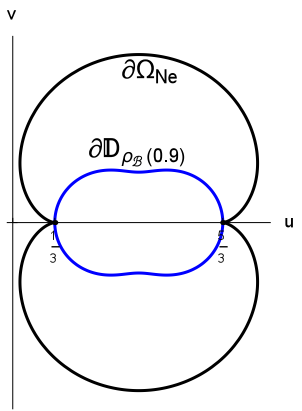

(b)

Figure 3. Sharpness of $\rho_{\mathcal{B}}(\alpha)$, where (a) $\alpha=0.1$ and (b) $\alpha=0.9$

Therefore,

$$
\mathcal{Q}_{f_{\mathcal{B}}}\left(\partial \mathbb{D}_{\rho_{\mathcal{B}}(\alpha)}\right) \cap \partial \Omega_{N e}=\{1 / 3,5 / 3\} \neq \emptyset .
$$

This proves that the estimate $\rho_{\mathcal{B}}(\alpha)$ is best possible. Figure 3 depicts the sharpness of $\rho_{\mathcal{B}}(\alpha)$ for different values of $\alpha$.

(ii): Let $f \in \mathcal{S}_{L}^{*}(\alpha)$. Then $\mathcal{Q}_{f}(z) \prec \alpha+(1-\alpha) \sqrt{1+z}$ and hence

$$
\begin{aligned}
\left|\mathcal{Q}_{f}(z)-1\right| & \leq|\alpha+(1-\alpha) \sqrt{1+z}-1| \\
& =(1-\alpha)|1-\sqrt{1+z}| \\
& \leq(1-\alpha)(1-\sqrt{1-r}), \quad|z|=r .
\end{aligned}
$$

An application of Lemma 2.1 shows that $f \in \mathcal{S}_{N e}^{*}$ if $(1-\alpha)(1-\sqrt{1-r}) \leq 2 / 3$, which on simplification gives $r \leq \rho_{L}(\alpha)$. Verify that the function

$$
f_{L}(z)=z+(1-\alpha) z^{2}+\frac{1}{16}(1-\alpha)(1-2 \alpha) z^{3}+\cdots
$$

satisfies $\mathcal{Q}_{f_{L}}(z)=\alpha+(1-\alpha) \sqrt{1+z}$, and hence is a member of $\mathcal{S}_{L}^{*}(\alpha)$. Moreover,

$$
\mathcal{Q}_{f_{L}}\left(z_{0}\right)=1 / 3 \in \partial \Omega_{N e}, \text { for } z_{0}=-\rho_{L}(\alpha) \in \partial \mathbb{D}_{\rho_{L}(\alpha)} .
$$

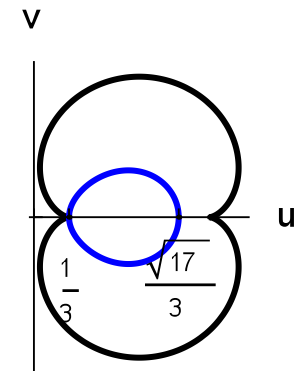

(a) $\alpha=0$

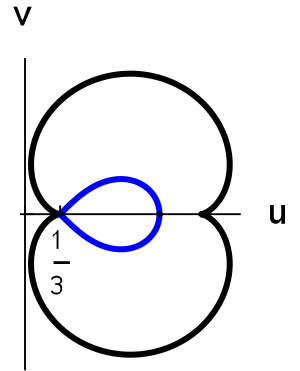

(b) $\alpha=1 / 3$

Figure 4. Sharpness of $\rho_{L}(\alpha)$

(iii): $f \in \mathcal{S}_{\alpha, e}^{*}$ implies $\mathcal{Q}_{f}(z) \prec \alpha+(1-\alpha) e^{z}$. For $|z|=r$, this subordination yields

$$
\left|\mathcal{Q}_{f}(z)-1\right| \leq(1-\alpha)\left|e^{z}-1\right| \leq(1-\alpha)\left(e^{r}-1\right) \leq 2 / 3
$$


if $r \leq \log ((5-3 \alpha) /(3-3 \alpha))=\rho_{e}(\alpha)$. Therefore, $f \in \mathcal{S}_{N e}^{*}$ if $|z|=r \leq \rho_{e}(\alpha)$. The result is sharp for the function $f_{e} \in \mathcal{S}_{\alpha, e}^{*}$ given by

$$
f_{e}(z)=z+(1-\alpha) z^{2}+\frac{1}{4}(1-\alpha)(3-2 \alpha) z^{3}+\cdots,
$$

and

$$
\mathcal{Q}_{f_{e}}\left(\partial \mathbb{D}_{\rho_{e}(\alpha)}\right) \cap \partial \Omega_{N e}=\{5 / 3\}
$$

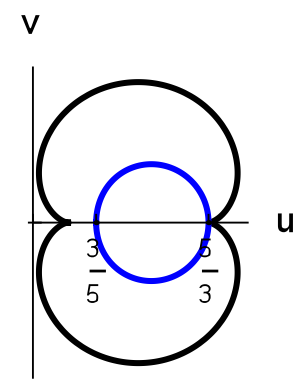

(a) $\alpha=0$

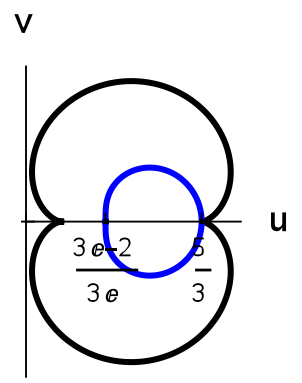

(b) $\alpha=(3 e-5) /(3 e-3)$

Figure 5. Sharpness of $\rho_{e}(\alpha)$

Theorem 3.3. For the function classes $\mathcal{S}_{R L}^{*}, \mathcal{S}_{C}^{*}$ and $\mathcal{S}_{R}^{*}$, we have:

(i) $R_{\mathcal{S}_{N e}^{*}}\left(\mathcal{S}_{R L}^{*}\right)=\rho_{R L}:=56 /(122-41 \sqrt{2}) \approx 0.874764$,

(ii) $R_{\mathcal{S}_{N e}^{*}}\left(\mathcal{S}_{C}^{*}\right)=\rho_{c}:=\sqrt{2}-1 \approx 0.414214$,

(iii) $R_{\mathcal{S}_{N e}^{*}}\left(\mathcal{S}_{R}^{*}\right)=\rho_{R}:=1 /(3 \sqrt{2}-3) \approx 0.804738$.

The estimate in each part is sharp (see Figure [).

Proof. (i): Let $f \in \mathcal{S}_{R L}^{*}$. Then $\mathcal{Q}_{f}(z) \prec \varphi_{R L}(z)$, where $\varphi_{R L}(z)$ is defined in (1.3). Thus, for $|z|=r<1$, we have

$$
\begin{aligned}
\left|\mathcal{Q}_{f}(z)-1\right| & \leq\left|\sqrt{2}-(\sqrt{2}-1) \sqrt{\frac{1-z}{1+2(\sqrt{2}-1) z}}-1\right| \\
& \leq 1-\left(\sqrt{2}-(\sqrt{2}-1) \sqrt{\frac{1+r}{1-2(\sqrt{2}-1) z}}\right) .
\end{aligned}
$$

In view of Lemma 2.1, the above disk lies inside the nephroid region $\Omega_{N e}$ provided

$$
1-\left(\sqrt{2}-(\sqrt{2}-1) \sqrt{\frac{1+r}{1-2(\sqrt{2}-1) z}}\right) \leq \frac{2}{3},
$$

Or equivalently, if $r \leq \rho_{R L}$. The result is sharp for the function $f_{R L} \in \mathcal{S}_{R L}^{*}$ given by

$$
\begin{aligned}
& f_{R L}(z)=z\left(\frac{\sqrt{1-z}+\sqrt{1+2(\sqrt{2}-1) z}}{2}\right)^{2 \sqrt{2}-2} \\
& \quad \times \exp \left(\sqrt{2(\sqrt{2}-1)} \tan ^{-1}(\Psi(z))\right),
\end{aligned}
$$


where

$$
\Psi(z)=\frac{\sqrt{2(\sqrt{2}-1)}(\sqrt{2(\sqrt{2}-1) z+1}-\sqrt{1-z})}{2(\sqrt{2}-1) \sqrt{1-z}+\sqrt{2(\sqrt{2}-1) z+1}} .
$$

Further, $\mathcal{Q}_{f_{R L}}\left(\partial \mathbb{D}_{\rho_{R L}}\right) \cap \partial \Omega_{N e}=\{1 / 3\}$.

(ii): $f \in \mathcal{S}_{C}^{*}$ implies the subordination $\mathcal{Q}_{f}(z) \prec 1+4 z / 3+2 z^{2} / 3$, which in turn gives

$$
\left|\mathcal{Q}_{f}(z)-1\right| \leq 2\left(r^{2}+2 r\right) / 3, \quad|z|=r .
$$

Applying Lemma 2.1, we conclude that $f \in \mathcal{S}_{N e}^{*}$ if $r^{2}+2 r \leq 1$, or, if $r \leq \sqrt{2}-1=\rho_{c}$. To verify the sharpness of the radius estimate $\rho_{c}$, consider the function

$$
f_{c}(z)=z \exp \left(\frac{4}{3} z+\frac{1}{3} z^{2}\right) \text {. }
$$

Observe that $f_{c} \in \mathcal{S}_{C}^{*}$, and

$$
\mathcal{Q}_{f_{c}}\left(z_{0}\right)=5 / 3 \in \partial \Omega_{N e}, \text { for } z_{0}=\sqrt{2}-1 \in \partial \mathbb{D}_{\rho_{c}} .
$$

Hence the result is sharp.

(iii): Let $f \in \mathcal{S}_{R}^{*}$. Then $\mathcal{Q}_{f}(z)-1 \prec z(k+z) / k(k-z)$, where $k=\sqrt{2}+1$. For $|z|=r$, this relation further implies

$$
\left|\mathcal{Q}_{f}(z)-1\right| \leq r(k+r) / k(k-r) \leq 2 / 3
$$

if $3 r^{2}+5 k r-2 k^{2} \leq 0$. This yields $r \leq \rho_{R}$. Thus, in view of Lemma 2.1, $f \in \mathcal{S}_{N e}^{*}$ for $|z| \leq \rho_{R}$. Now consider the function

$$
f_{R}(z)=\frac{k^{2} z}{(k-z)^{2}} e^{-z / k}, \quad k=\sqrt{2}+1 .
$$

Simple calculations show that $\mathcal{Q}_{f_{R}}(z)=\left(k^{2}+z^{2}\right) / k(k-z)$, and hence $f_{R} \in \mathcal{S}_{R}^{*}$. Moreover, the value of $\mathcal{Q}_{f_{R}}(z)$ at the point $z=\rho_{R} \in \partial \mathbb{D}_{\rho_{R}}$ is $5 / 3$, i.e.,

$$
\mathcal{Q}_{f_{R}}\left(\partial \mathbb{D}_{\rho_{R}}\right) \cap \partial \Omega_{N e}=\{5 / 3\} \neq \emptyset .
$$

Hence the estimate is best possible.

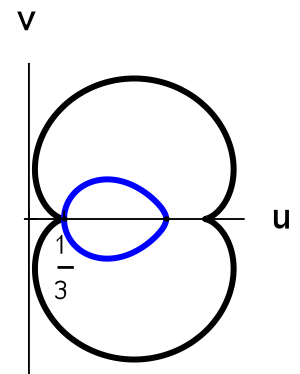

(a) Sharpness of $\rho_{R L}$

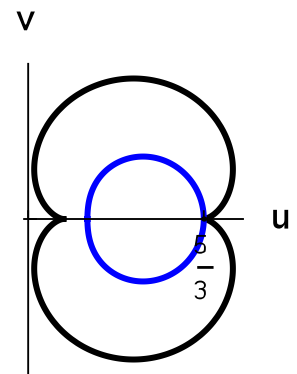

(b) Sharpness of $\rho_{c}$

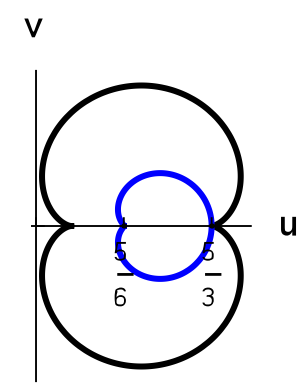

(c) Sharpness of $\rho_{R}$

Figure 6

Theorem 3.4. The $\mathcal{S}_{N e}^{*}$-radii for the classes $\mathcal{S}_{\mathbb{\beta}}^{*}$ and $\mathcal{S}_{S}^{*}$ are given by

(i) $R_{\mathcal{S}_{N e}^{*}}\left(\mathcal{S}_{\overparen{\zeta}}^{*}\right)=(\sqrt{17}-1) / 6 \approx 0.520518$,

(ii) $R_{\mathcal{S}_{N e}^{*}}\left(\mathcal{S}_{S}^{*}\right)=\sinh ^{-1}(2 / 3)=\log ((\sqrt{13}+2) / 3) \approx 0.625145$.

The estimates are not sharp. 


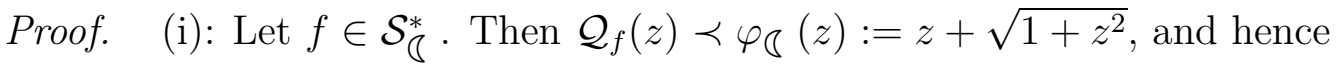

$$
\left|\mathcal{Q}_{f}(z)-1\right| \leq\left|z+\sqrt{1+z^{2}}-1\right| \leq 1+r-\sqrt{1-r^{2}}, \quad|z|=r<1 .
$$

From Lemma 2.1, it follows that the disk (3.3) lies in the interior of the nephroid region $\Omega_{N e}$ if $1+r-\sqrt{1-r^{2}} \leq 2 / 3$, or, if $r^{2}+r / 3-4 / 9 \leq 0$. This inequality gives the desired radius estimate. We note that a graphical observation, as well as the fact

$$
\varphi_{\zeta}(|z|=(\sqrt{17}-1) / 6) \cap \partial \Omega_{N e}=\emptyset,
$$

shows that the estimate is not sharp.

(ii): If $f \in \mathcal{S}_{S}^{*}$, then $\mathcal{Q}_{f}(z) \prec 1+\sin z$. For, $|z|=r<1$, this yields

$$
\left|\mathcal{Q}_{f}(z)-1\right| \leq|\sin z| \leq \sinh r \leq 2 / 3
$$

provided $r \leq \sinh ^{-1}(2 / 3)$. The desired result follows by an application of Lemma 2.1 . Again, the result is non-sharp.

In view of the above theorem, the following problem is posed.

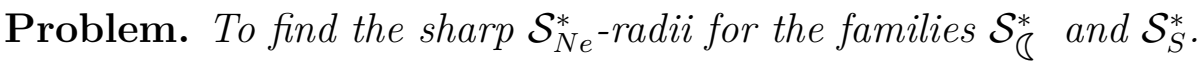

\section{4. $\mathcal{S}_{N e, n}^{*}$-Radius For Classes Defined as Ratio of Functions} form

Let $n \in \mathbb{N}:=\{1,2,3, \ldots\}$. Let $\mathcal{A}_{n}$ be the collection of all analytic functions $f(z)$ of the

$$
f(z)=z+\sum_{k=n+1}^{\infty} a_{k} z^{k}
$$

For $0 \leq \alpha<1$, let $\mathcal{P}_{n}(\alpha)$ consists of analytic functions $p(z)=1+\sum_{k=n}^{\infty} p_{k} z^{k}$ satisfying $\operatorname{Re}(p(z))>\alpha, z \in \mathbb{D}$. Further, let

$$
\mathcal{S}_{N e, n}^{*}:=\mathcal{S}_{N e}^{*} \cap \mathcal{A}_{n}, \mathcal{S}_{n}^{*}:=\mathcal{S}^{*} \cap \mathcal{A}_{n}, \mathcal{C}_{n}:=\mathcal{C} \cap \mathcal{A}_{n} \text { and } \mathcal{P}_{n}:=\mathcal{P}_{n}(0) .
$$

In this section, we will find the $\mathcal{S}_{N e, n}^{*}$-radius for several families defined as the ratio of certain special analytic functions. In the first theorem, we consider the following four families. For details, we refer [2].

$$
\begin{gathered}
\mathcal{G}_{1}:=\left\{f \in \mathcal{A}_{n}: \frac{f}{g} \in \mathcal{P}_{n} \text { and } \frac{g(z)}{z} \in \mathcal{P}_{n}, g \in \mathcal{A}_{n}\right\}, \\
\mathcal{G}_{2}:=\left\{f \in \mathcal{A}_{n}: \frac{f}{g} \in \mathcal{P}_{n} \text { and } \frac{g(z)}{z} \in \mathcal{P}_{n}\left(\frac{1}{2}\right), g \in \mathcal{A}_{n}\right\}, \\
\mathcal{G}_{3}:=\left\{f \in \mathcal{A}_{n}:\left|\frac{f(z)}{g(z)}-1\right|<1 \text { and } \frac{g(z)}{z} \in \mathcal{P}_{n}, g \in \mathcal{A}_{n}\right\},
\end{gathered}
$$

and

$$
\mathcal{G}_{4}:=\left\{f \in \mathcal{A}_{n}:\left|\frac{f(z)}{g(z)}-1\right|<1, g \in \mathcal{C}_{n}\right\} .
$$

To prove the results, we make use of the following combined lemma.

Lemma $4.1([18],[20])$. Let $p \in \mathcal{P}_{n}(\alpha)$. Then, for $|z|=r$, the following results hold. 
(a) (cf. [18, Lemma 2.1])

$$
\left|p(z)-\frac{1+(1-2 \alpha) r^{2 n}}{1-r^{2 n}}\right| \leq \frac{2(1-\alpha) r^{n}}{1-r^{2 n}} .
$$

(b) (cf. [20, Lemma 2])

$$
\left|\frac{z p^{\prime}(z)}{p(z)}\right| \leq \frac{2 n r^{n}(1-\alpha)}{\left(1-r^{n}\right)\left(1+(1-2 \alpha) r^{n}\right)} .
$$

Theorem 4.1. For the function classes $\mathcal{G}_{1}, \mathcal{G}_{2}, \mathcal{G}_{3}$ and $\mathcal{G}_{4}$, we have the following radii results.

(i) $R_{\mathcal{S}_{N e, n}^{*}}\left(\mathcal{G}_{1}\right)=\rho_{1}:=\left(3 n+\sqrt{9 n^{2}+1}\right)^{-1 / n}$.

(ii) $R_{\mathcal{S}_{N e, n}^{*}}\left(\mathcal{G}_{2}\right)=\rho_{2}:=\sqrt[n]{4} \times\left(9 n+\sqrt{81 n^{2}+24 n+16}\right)^{-1 / n}$.

(iii) $R_{\mathcal{S}_{N e, n}^{*}}\left(\mathcal{G}_{3}\right)=\rho_{3}:=\sqrt[n]{4} \times\left(9 n+\sqrt{(4+9 n)^{2}-48 n}\right)^{-1 / n}$.

(iv) $R_{\mathcal{S}_{N e, n}^{*}}\left(\mathcal{G}_{4}\right)=\rho_{4}:=\sqrt[n]{4} \times\left(3(n+1)+\sqrt{(1+3 n)^{2}+36 n}\right)^{-1 / n}$.

Each estimate is sharp.

Proof. (i): Let $f \in \mathcal{G}_{1}$. Further, suppose that

$$
p(z)=g(z) / z \quad \text { and } \quad q(z)=f(z) / g(z) .
$$

Clearly, $p, q \in \mathcal{P}_{n}$. Thus, for $|z|=r$, the following inequalities follow from Lemma 4.1((b)

$$
\left|\frac{z p^{\prime}(z)}{p(z)}\right| \leq \frac{2 n r^{n}}{1-r^{2 n}} \quad \text { and } \quad\left|\frac{z q^{\prime}(z)}{q(z)}\right| \leq \frac{2 n r^{n}}{1-r^{2 n}} .
$$

Moreover, we have $f(z)=g(z) q(z)=z p(z) q(z)$, which gives the identity

$$
\mathcal{Q}_{f}(z)=\frac{z f^{\prime}(z)}{f(z)}=1+\frac{z p^{\prime}(z)}{p(z)}+\frac{z q^{\prime}(z)}{q(z)} .
$$

Using the inequalities (4.1), the above identity yields the disk

$$
\left|\mathcal{Q}_{f}(z)-1\right| \leq 4 n r^{n} /\left(1-r^{2 n}\right) .
$$

In view of Lemma 2.1, the quantity $\mathcal{Q}_{f}(z)$ takes values from the region bounded by the nephroid (1.4) provided $4 n r^{n} /\left(1-r^{2 n}\right) \leq 2 / 3$, or equivalently, $r^{2 n}+6 n r^{n}-1 \leq 0$. This further gives $r \leq \rho_{1}$. To verify the sharpness of the bound $\rho_{1}$, consider the functions $f_{1}, g_{1} \in \mathcal{A}_{n}$ given by

$$
f_{1}(z)=z\left(\frac{1+z^{n}}{1-z^{n}}\right)^{2} \quad \text { and } \quad g_{1}(z)=z\left(\frac{1+z^{n}}{1-z^{n}}\right) .
$$

It is clear that $f_{1}(z) / g_{1}(z)=g_{1}(z) / z=\left(1+z^{n}\right) /\left(1-z^{n}\right) \in \mathcal{P}_{n}$, and hence $f_{1} \in \mathcal{G}_{1}$. Also,

$$
\mathcal{Q}_{f_{1}}(z)=1+4 n z^{n} /\left(1-z^{2 n}\right),
$$

and at the points $z= \pm \rho_{1} \in \partial \mathbb{D}_{\rho_{1}}$, we have

$$
\mathcal{Q}_{f_{1}}\left(-\rho_{1}\right)=1 / 3 \in \partial \Omega_{N e} \text { and } \mathcal{Q}_{f_{1}}\left(\rho_{1}\right)=5 / 3 \in \partial \Omega_{N e} .
$$

This proves sharpness of the estimate. 
(ii): Let $f \in \mathcal{G}_{2}$. Define the functions $p, q: \mathbb{D} \rightarrow \mathbb{C}$ by $p=g / z$ and $q=f / g$. Then $f(z)=z p(z) q(z)$ with $p \in \mathcal{P}_{n}(1 / 2)$ and $q \in \mathcal{P}_{n}$. In light of the identity (4.2), it follows from Lemma $4.1(\mathrm{~b})$ that

$$
\left|\mathcal{Q}_{f}(z)-1\right| \leq \frac{2 n r^{n}}{1-r^{2 n}}+\frac{n r^{n}}{1-r^{n}}=\frac{3 n r^{n}+n r^{2 n}}{1-r^{2 n}} .
$$

Now, from Lemma 2.1, $f \in \mathcal{S}_{N e}^{*}$ if

$$
\frac{3 n r^{n}+n r^{n}}{1-r^{2 n}} \leq \frac{2}{3}
$$

That is, if $(3 n+2) r^{2 n}+9 n r^{n}-2 \leq 0$, which gives the desired result $r \leq \rho_{2}$. To prove that $\rho_{2}$ is the sharp $\mathcal{S}_{N e, n}^{*}$-radius for the function family $\mathcal{G}_{2}$, define

$$
f_{2}(z)=\frac{z\left(1+z^{n}\right)}{\left(1-z^{n}\right)^{2}} \quad \text { and } \quad g_{2}(z)=\frac{z}{1-z^{n}} .
$$

It is easy to see that $f_{2} / g_{2} \in \mathcal{P}_{n}$ and $g_{2} / z \in \mathcal{P}_{n}(1 / 2)$, which shows that $f_{2}$ is a member of $\mathcal{G}_{2}$. Further,

$$
\left.\mathcal{Q}_{f_{2}}(z)\right|_{z=\rho_{2}}=\left.\frac{1+3 n z^{n}+(n-1) z^{2 n}}{1-z^{2 n}}\right|_{z=\rho_{2}}=\frac{5}{3} .
$$

This shows that

$$
\mathcal{Q}_{f_{2}}\left(\partial \mathbb{D}_{\rho_{2}}\right) \cap \partial \Omega_{N e}=\{5 / 3\} \neq \emptyset,
$$

and hence the estimate is sharp.

(iii): Let $f \in \mathcal{G}_{3}$. Further, suppose that $p(z)=g(z) / z$ and $q(z)=g(z) / f(z)$. Obviously $p \in \mathcal{P}_{n}$, and $q \in \mathcal{P}_{n}(1 / 2)$ due to the fact that

$$
\left|\frac{f(z)}{g(z)}-1\right|<1 \Longleftrightarrow \frac{g}{f} \in \mathcal{P}_{n}\left(\frac{1}{2}\right) \text {. }
$$

Verify that $f(z)=z p(z) / q(z)$ and

$$
\mathcal{Q}_{f}(z)=1+\frac{z p^{\prime}(z)}{p(z)}-\frac{z q^{\prime}(z)}{q(z)} .
$$

Applying Lemma 4.1(b) and simplifying, we obtain

$$
\left|\mathcal{Q}_{f}(z)-1\right| \leq\left(3 n r^{n}+n r^{2 n}\right) /\left(1-r^{2 n}\right) \leq 2 / 3
$$

if $(3 n+2) r^{2 n}+9 n r^{n}-2 \leq 0$, or, $r \leq \rho_{3}$. Therefore, by Lemma 2.1, $f \in \mathcal{S}_{N e}^{*}$ provided $r \leq \rho_{3}$. For sharpness, consider

$$
f_{3}(z)=\frac{z\left(1+z^{n}\right)^{2}}{1-z^{n}} \quad \text { and } \quad g_{3}(z)=\frac{z\left(1+z^{n}\right)}{1-z^{n}}
$$

satisfying

$$
\left|\frac{f_{3}(z)}{g_{3}(z)}-1\right|=|z|^{n}<1 \quad \text { and } \quad \frac{g_{3}(z)}{z}=\frac{1+z^{n}}{1-z^{n}} \in \mathcal{P}_{n},
$$

so that $f_{3} \in \mathcal{G}_{3}$. Moreover, it is easy to see that $\mathcal{Q}_{f_{3}}(z)$ assumes the value $1 / 3$ at the point $z=\rho_{3} e^{i \pi / n} \in \partial \mathbb{D}_{\rho_{3}}$. This shows that the estimate is best possible. 
(iv): Let $f \in \mathcal{G}_{4}$ and let $q: \mathbb{D} \rightarrow \mathbb{C}$ be given by $q=g / f$, where $g \in \mathcal{A}_{n}$ is some convex

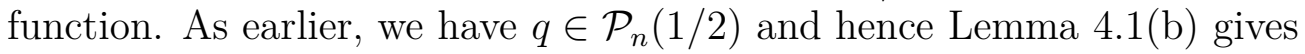

$$
\left|\frac{z q^{\prime}(z)}{q(z)}\right| \leq \frac{n r^{n}}{1-r^{n}}
$$

Further, since convexity of $g$ implies $z g^{\prime} / g \in \mathcal{P}_{n}(1 / 2)$, we have the following inequality from Lemma 4.1(国),

$$
\left|\frac{z g^{\prime}(z)}{g(z)}-\frac{1}{1-r^{2 n}}\right| \leq \frac{r^{n}}{1-r^{2 n}}
$$

In view of the inequalities (4.3) and (4.4), and the identity

$$
\mathcal{Q}_{f}(z)=\frac{z g^{\prime}(z)}{g(z)}-\frac{z q^{\prime}(z)}{q(z)},
$$

we calculate that

$$
\left|\mathcal{Q}_{f}(z)-\frac{1}{1-r^{2 n}}\right| \leq \frac{(n+1) r^{n}+n r^{2 n}}{1-r^{2 n}} .
$$

As the number $1-r^{2 n}<1$ for each $r \in(0,1)$, it follows from Lemma 2.1 that the disc (4.5) lies in the interior of the nephroid domain $\Omega_{N e}$ if

$$
\frac{(n+1) r^{n}+n r^{2 n}}{1-r^{2 n}} \leq \frac{1}{1-r^{2 n}}-\frac{1}{3} \text {. }
$$

Or equivalently, if

$$
(3 n-1) r^{2 n}+3(n+1) r^{n}-2 \leq 0 .
$$

This further gives $r \leq \rho_{4}$. For sharpness of the estimate, consider

$$
f_{4}(z)=\frac{z\left(1+z^{n}\right)}{\left(1-z^{n}\right)^{1 / n}} \quad \text { and } \quad g_{4}(z)=\frac{z}{\left(1-z^{n}\right)^{1 / n}} .
$$

Since $\left|f_{4}(z) / g_{4}(z)-1\right|=|z|^{n}<1$ and $g_{4} \in \mathcal{C}_{n}$, we have $f_{4} \in \mathcal{G}_{4}$. Moreover, $\mathcal{Q}_{f_{4}}\left(-\rho_{4}\right)=1 / 3$ and $\mathcal{Q}_{f_{4}}\left(\rho_{4}\right)=5 / 3$, i,e.,

$$
\mathcal{Q}_{f_{4}}\left(\partial \mathbb{D}_{\rho_{4}}\right) \cap \partial \Omega_{N e}=\{1 / 3,5 / 3\} \neq \emptyset \text {. }
$$

Therefore, the estimate is sharp.

In continuation of our discussion, we provide radii results for two classes of functions that possess nice geometrical properties. These classes had less attention with respect to properties other than the radii results, after the celebrated de Branges's theorem.

The close-to-starlike class $\mathcal{C S}_{n}^{*}(\alpha)$. For $\alpha \in[0,1)$, the class of close-to-starlike functions $\mathcal{C S}_{n}^{*}(\alpha)$ of type $\alpha$ was introduced by Reade [19] and is defined as

$$
\mathcal{C S}^{*}(\alpha):=\left\{f \in \mathcal{A}_{n}: \frac{f}{g} \in \mathcal{P}_{n}, g \in \mathcal{S}_{n}^{*}(\alpha)\right\} .
$$

The following theorem determines the $\mathcal{S}_{N e, n}^{*}$-radius for $\mathcal{C S}_{n}^{*}(\alpha)$.

Theorem 4.2. The sharp $\mathcal{S}_{N e, n}^{*}$-radius for $\mathcal{C S}_{n}^{*}(\alpha)$ is given by

$$
R_{\mathcal{S}_{N e, n}^{*}}\left(\mathcal{C S}_{n}^{*}(\alpha)\right)=\rho_{c s}:=\left(\frac{2}{3(1+n-\alpha)+\sqrt{9(1+n-\alpha)^{2}+4(4-3 \alpha)}}\right)^{1 / n} .
$$


Proof. If $f \in \mathcal{C S}_{n}^{*}(\alpha)$, then $h=f / g \in \mathcal{P}_{n}$ for some function $g \in \mathcal{S}_{n}^{*}(\alpha)$. Applying Lemma 4.1(b), we have

$$
\left|\frac{z h^{\prime}(z)}{h(z)}\right| \leq \frac{2 n r^{n}}{1-r^{2 n}}
$$

Also, $g \in \mathcal{S}_{n}^{*}(\alpha)$ implies $z g^{\prime} / g \in \mathcal{P}_{n}(\alpha)$, so that Lemma 4.1(国) gives

$$
\left|\frac{z g^{\prime}(z)}{g(z)}-\frac{1+(1-2 \alpha) r^{2 n}}{1-r^{2 n}}\right| \leq \frac{2(1-\alpha) r^{n}}{1-r^{2 n}}
$$

In view of the identity

$$
\mathcal{Q}_{f}(z)=\frac{z g^{\prime}(z)}{g(z)}+\frac{z h^{\prime}(z)}{h(z)},
$$

it follows from (4.6) and (4.7) that

$$
\left|\mathcal{Q}_{f}(z)-\frac{1+(1-2 \alpha) r^{2 n}}{1-r^{2 n}}\right| \leq \frac{2(1+n-\alpha) r^{n}}{1-r^{2 n}} .
$$

The inequality (4.8) represents a disk with center $a$ and radius $r_{0}$ given by

$$
a:=\frac{1+(1-2 \alpha) r^{2 n}}{1-r^{2 n}} \text { and } r_{0}:=\frac{2(1+n-\alpha) r^{n}}{1-r^{2 n}} .
$$

As $a>1$, we conclude from Lemma 2.1 that the disk (4.8) lies in the interior of the region $\Omega_{N e}$ bounded by the nephroid (1.4) if $r_{0} \leq 5 / 3-a$. That is, if

$$
(4-3 \alpha) r^{2 n}+3(1+n-\alpha) r^{n}-1 \leq 0 .
$$

On solving this inequality we obtain $r \leq \rho_{c s}$. For sharpness, consider the functions $f, g \in \mathcal{A}_{n}$ given by

$$
f(z)=\frac{z\left(1+z^{n}\right)}{\left(1-z^{n}\right)^{(n+2-2 \alpha) / n}} \quad \text { and } \quad g(z)=\frac{z}{\left(1-z^{n}\right)^{(2-2 \alpha) / n}} .
$$

Since $f / g=\left(1+z^{n}\right) /\left(1-z^{n}\right) \in \mathcal{P}_{n}$ and $g \in \mathcal{S}_{n}^{*}(\alpha)$, the function $f \in \mathcal{C S}_{n}^{*}(\alpha)$. For this function $f$, we have $\mathcal{Q}_{f}\left(\rho_{c s}\right)=5 / 3$. Thus the estimate is sharp.

The MacGregor class $\mathcal{W}_{n}$. Define the class $\mathcal{W}_{n}$ as

$$
\mathcal{W}_{n}:=\left\{f \in \mathcal{A}_{n}: f / z \in \mathcal{P}_{n}\right\} \text {. }
$$

Introduced first by MacGregor [13, this function class was recently studied for several radius problems by the authors in [1, 15, 14, 15]. Following them, we prove the following sharp radius result for $\mathcal{W}_{n}$.

Theorem 4.3. The sharp $\mathcal{S}_{N e, n}^{*}$-radius for $\mathcal{W}_{n}$ is

$$
R_{\mathcal{S}_{N e, n}^{*}}\left(\mathcal{W}_{n}\right)=\rho_{\mathcal{W}}:=\left(2 /\left(3 n+\sqrt{9 n^{2}+4}\right)\right)^{1 / n} .
$$

Proof. Let $f \in \mathcal{W}_{n}$ and $|z|=r<1$. Then $h(z)=f(z) / z \in \mathcal{P}_{n}$ and Lemma 4.1(b) gives

$$
\left|\frac{z h^{\prime}(z)}{h(z)}\right| \leq \frac{2 n r^{n}}{1-r^{2 n}}
$$

On using the above inequality in the identity $\mathcal{Q}_{f}(z)=1+z h^{\prime}(z) / h(z)$, we obtain

$$
\left|\mathcal{Q}_{f}(z)-1\right| \leq 2 n r^{n} /\left(1-r^{2 n}\right) .
$$

Now applying Lemma 2.1, it follows that $\mathcal{Q}_{f} \in \Omega_{N e}$ if $2 n r^{n} /\left(1-r^{2 n}\right) \leq 2 / 3$, or, if $r^{2 n}+3 n r^{n}-1 \leq 0$. The last inequality yields $r \leq \rho_{\mathcal{M}}$. For the function $f_{\mathcal{W}}(z)=$ 
$z\left(1+z^{n}\right) /\left(1-z^{n}\right)$ satisfying $f(z) / z \in \mathcal{P}_{n}$, we have $\mathcal{Q}_{f_{\mathcal{W}}}\left(-\rho_{\mathcal{W}}\right)=1 / 3$ and $\mathcal{Q}_{f_{\mathcal{W}}}\left(\rho_{\mathcal{W}}\right)=5 / 3$. Therefore,

$$
\mathcal{Q}_{f_{\mathcal{W}}}\left(\partial \mathbb{D}_{\rho_{\mathcal{W}}}\right) \cap \partial \Omega_{N e}=\{1 / 3,5 / 3\} \neq \emptyset
$$

and hence the estimate is best possible.

Besides the above mentioned two classes, similar type of radius results for various other classes can be obtained. Among these, as an exhibit, the authors favor the following class.

The class $\mathcal{M}_{n}(\beta)$. For $\beta>1$, the family of functions $\mathcal{M}_{n}(\beta)$ introduced by Uralegaddi et al. [26] is defined as

$$
\mathcal{M}_{n}(\beta):=\left\{f \in \mathcal{A}_{n}: \operatorname{Re}\left(\mathcal{Q}_{f}(z)\right)<\beta, z \in \mathbb{D}\right\} .
$$

In terms of subordination, $\mathcal{M}_{n}(\beta)$ has the following characterization:

$$
f \in \mathcal{M}_{n}(\beta) \Longleftrightarrow \mathcal{Q}_{f}(z) \prec \frac{1+(1-2 \beta) z^{n}}{1-z^{n}}, \quad \beta>1 .
$$

The class $\mathcal{M}_{n}(\beta)$ gained less attention from the researchers working in this direction. Nevertheless, it compeers the same significance, in analytic properties, as that of the class of starlike functions with negative coefficients introduced by Silverman [22]. For this interesting class of functions, we have the following result without details of the proof, as the proof can be traced in lines similar to the proof of Theorem 4.2.

Theorem 4.4. The $\mathcal{S}_{N e, n}^{*}$-radius for the class $\mathcal{M}_{n}(\beta)$ is given by

$$
R_{\mathcal{S}_{N e, n}^{*}}\left(\mathcal{M}_{n}(\beta)\right)=(3 \beta-2)^{-1 / n} .
$$

The result is sharp for the function $f(z)=z\left(1-z^{n}\right)^{2(\beta-1) / n}$.

\section{REFERENCES}

[1] R. M. Ali, N. K. Jain and V. Ravichandran, Radii of starlikeness associated with the lemniscate of Bernoulli and the left-half plane, Appl. Math. Comput. 218 (2012), no. 11, 6557-6565.

[2] R. M. Ali, N. K. Jain and V. Ravichandran, On the radius constants for classes of analytic functions, Bull. Malays. Math. Sci. Soc. (2) 36 (2013), no. 1, 23-38.

[3] Y.-L. Cang and J.-L. Liu, Radius of convexity for certain analytic functions associated with the lemniscate of Bernoulli, Expo. Math. 33 (2015), no. 3, 387-391.

[4] N. E. Cho, S. Kumar, V. Kumar and V. Ravichandran, Differential subordination and radius estimates for starlike functions associated with the Booth lemniscate, Turkish J. Math. 42 (2018), no. 3, 1380-1399.

[5] N. E. Cho, V. Kumar, S. S. Kumar and V. Ravichandran, Radius problems for starlike functions associated with the sine function, Bull. Iranian Math. Soc. 45 (2019), no. 1, 213-232.

[6] S. Gandhi and V. Ravichandran, Starlike functions associated with a lune, Asian-Eur. J. Math. 10 (2017), no. 4, 1750064, 12 pp.

[7] A. W. Goodman, Univalent functions. Vol. II, Mariner Publishing Co., Inc., Tampa, FL, 1983.

[8] W. Janowski, Some extremal problems for certain families of analytic functions. I, Ann. Polon. Math. 28 (1973), 297-326.

[9] R. Kargar, A. Ebadian and J. Sokół, On Booth lemniscate and starlike functions, Anal. Math. Phys. 9 (2019), no. 1, 143-154.

[10] K. Khatter, V. Ravichandran and S. Sivaprasad Kumar, Starlike functions associated with exponential function and the lemniscate of Bernoulli, Rev. R. Acad. Cienc. Exactas Fís. Nat. Ser. A Mat. RACSAM 113 (2019), no. 1, 233-253.

[11] S. Kumar and V. Ravichandran, A subclass of starlike functions associated with a rational function, Southeast Asian Bull. Math. 40 (2016), no. 2, 199-212.

[12] W. C. Ma and D. Minda, A unified treatment of some special classes of univalent functions, in Proceedings of the Conference on Complex Analysis (Tianjin, 1992), 157-169, Conf. Proc. Lecture Notes Anal., I, Int. Press, Cambridge. 
[13] T. H. MacGregor, The radius of univalence of certain analytic functions, Proc. Amer. Math. Soc. 14 (1963), 514-520.

[14] R. Mendiratta, S. Nagpal and V. Ravichandran, A subclass of starlike functions associated with left-half of the lemniscate of Bernoulli, Internat. J. Math. 25 (2014), no. 9, 1450090, 17 pp.

[15] R. Mendiratta, S. Nagpal and V. Ravichandran, On a subclass of strongly starlike functions associated with exponential function, Bull. Malays. Math. Sci. Soc. 38 (2015), no. 1, 365-386.

[16] S. S. Miller and P. T. Mocanu, Differential subordinations, Monographs and Textbooks in Pure and Applied Mathematics, 225, Marcel Dekker, Inc., New York, 2000.

[17] R. K. Raina and J. Sokół, Some properties related to a certain class of starlike functions, C. R. Math. Acad. Sci. Paris 353 (2015), no. 11, 973-978.

[18] V. Ravichandran, F. Rønning and T. N. Shanmugam, Radius of convexity and radius of starlikeness for some classes of analytic functions, Complex Variables Theory Appl. 33 (1997), no. 1-4, 265-280.

[19] M. O. Reade, On close-to-convex univalent functions, Michigan Math. J. 3 (1955), 59-62.

[20] G. M. Shah, On the univalence of some analytic functions, Pacific J. Math. 43 (1972), 239-250.

[21] K. Sharma, N. K. Jain and V. Ravichandran, Starlike functions associated with a cardioid, Afr. Mat. 27 (2016), no. 5-6, 923-939.

[22] H. Silverman, Univalent functions with negative coefficients, Proc. Amer. Math. Soc. 51 (1975), $109-116$.

[23] J. Sokół and J. Stankiewicz, Radius of convexity of some subclasses of strongly starlike functions, Zeszyty Nauk. Politech. Rzeszowskiej Mat. No. 19 (1996), 101-105.

[24] J. Sokól, Radius problems in the class $S L^{*}$, Appl. Math. Comput. 214 (2009), no. 2, 569-573.

[25] J. Sokół and D. K. Thomas, Further results on a class of starlike functions related to the Bernoulli lemniscate, Houston J. Math. 44 (2018), no. 1, 83-95.

[26] B. A. Uralegaddi, M. D. Ganigi and S. M. Sarangi, Univalent functions with positive coefficients, Tamkang J. Math. 25 (1994), no. 3, 225-230.

[27] L. A. Wani and A. Swaminathan, Starlike and convex functions associated with a nephroid domain, arXiv:1912.05767, (2019), 23 pages.

${ }^{\dagger}$ Department of Mathematics, Indian Institute of Technology, Roorkee-247667, UtTARAKHAND, INDIA

E-mail address: lateef17304@gmail.com

‡Department of Mathematics, Indian Institute of Technology, Roorkee-247667, UtTARAKHAND, INDIA

E-mail address: mathswami@gmail.com, a.swaminathan@ma.iitr.ac.in 